\title{
Pathway landscapes and epigenetic regulation in breast cancer and melanoma cell lines
}

\author{
Mariama El Baroudi ${ }^{1}$, Dario La Sala ${ }^{2}$, Caterina Cinti ${ }^{3}$, Enrico Capobianco ${ }^{1,4^{*}}$ \\ From 1st International Work-Conference on Bioinformatics and Biomedical Engineering-IWBBIO 2013 \\ Granada, Spain. 18-20 March 2013
}

\author{
* Correspondence: \\ ECapobianco@med.miami.edu \\ ${ }^{1}$ Laboratory of Integrative Systems \\ Medicine (LISM), Institute of Clinical \\ Physiology (IFC) \& Institute of \\ Informatics and Telematics (IIT), \\ National Research Council (CNR), \\ Pisa, 56124, Italylgnacio Rojas and \\ Francisco Ortuño
}

\begin{abstract}
Background: Epigenetic variation is a main regulation mechanism of gene expression in various cancer histotypes, and due to its reversibility, the potential impact in therapy can be very relevant.

Methods: Based on a selected pair, breast cancer (BC) and melanoma, we conducted inference analysis in parallel on a few cell lines (MCF-7 for BC and A375

for melanoma). Starting from differential expression after treatment with a demethylating agent, the 5-Aza-2'-deoxycytidine (DAC), we provided pathway enrichment analysis and gene regulatory maps with cross-linked microRNAs and transcription factors.

Results: Several oncogenic signaling pathways altered upon DAC treatment were detected with significant enrichment. We represented the association between these cancers by depicting the landscape of common and specific variation affecting them.
\end{abstract}

\section{Introduction}

Recent advances in the field of epigenetics have provided new insights on the global epigenetic modifications that promote cancer development and progression [1,2]. Notably, epigenetic therapy, which is a consequence of the reversible nature of the epigenetic changes that alter gene expression in many tumor histotypes [3,4], is the ultimate interest of many proposed research projects, including the present one. Among several drugs with antitumorigenic effect regulating the epigenetic status of cells, 5-Aza-2'-deoxycytidine (DAC, Dagogen) is a potent demethylating agent known for anti-leukemic effect in the mouse model [5,6]. DAC acts to correct epigenetic defect including reactivation of tumor suppressor genes (TSG) [7] silenced by epigenetic mechanisms in tumor tissues [2]. Combining DAC with a chemotherapeutic agent (Carboplatin) in patients with recurrent, platinum-resistant, Epithelial Ovarian Cancer (EOC), was shown to exert a potent demethylating effect justifying the need of further testing for clinical efficacy [8]. The DAC effect was evaluated in breast cancer (BC) cell lines by gene expression analysis: at the used concentrations, there is a role appearing for treatment in different cellular processes linked to TNF- $\alpha$-dependent apoptosis [9]. Also, the safety of DAC combined with chemotherapy in metastatic melanoma was reported in [10].

C 2014 Baroudi et al.: licensee BioMed Central Ltd. This is an Open Access article distributed under the terms of the Creative Commons Attribution License (http://creativecommons.org/licenses/by/2.0), which permits unrestricted use, distribution, and reproduction in any medium, provided the original work is properly cited. The Creative Commons Public Domain Dedication waiver (http:// creativecommons.org/publicdomain/zero/1.0/) applies to the data made available in this article, unless otherwise stated. 
Rationale for the association between $\mathrm{BC}$ and melanoma, is provided first of all by evidence: genetic relationships and common variant genes appeared in [11-13]; a high risk association of melanoma in $\mathrm{BC}$ patients was reported in [14]; this risk holds especially for patients not receiving anti-estrogen therapy [15]; several biomarkers have been proposed to identify cancer stem cells in these two tumors [16]. In general, risk factors such as family history play a role in both cancers. Moreover, carriers of mutations in BRCA2 - the $\mathrm{BC}$ predisposition gene - have an increased risk of melanoma, while carriers of mutations in the melanoma susceptibility gene - CDKN2A - exhibit a higher than expected risk of $\mathrm{BC}$. We therefore hypothesized that pathways involved in the development of both cancers may to a certain extent overlap, and that survivors of one cancer may be prone to develop the other one. Specifically, epigenetic mechanisms which sum to mutations during cancer progression, can be explored through pathways that are in common between the two cancers and result up-regulated after DAC treatment.

Our goal is to investigate DAC treatment effects in MCF-7 (BC) and A375 (melanoma) cell lines. We elucidate pathway landscapes and regulatory networks following gene expression profiling and aimed to identify epigenetically modified genes. Once the functionally enriched pathways are identified, both transcriptional and post-transcriptional regulatory networks are derived with the ultimate goal of assigning to them a role of possible drivers of future developments in novel anticancer target therapies. The sections of the paper are organized into Methods, Results, and Discussion at the end.

\section{Methods}

\section{Microarray analysis}

The MCF-7 and A375 cell lines were cultured in DMEM medium supplemented with 10\% fetal bovine serum, $2 \mathrm{mM} \mathrm{L-glutamine,} \mathrm{at} \mathrm{split} \mathrm{ratio} \mathrm{of} \mathrm{1:4} \mathrm{twice} \mathrm{a} \mathrm{week.} \mathrm{After} 24$ hours of spit the culture medium was changed with media containing 2,5 $\mu \mathrm{M}$ 5-Aza-2-dC (DAC). 5-aza-2'-deoxcytidine (Sigma) was prepared freshly prior of experiments as a $10 \mathrm{mM}$ stock solution diluted in acetic acid: water (1:1). The treated cells were collected after 48 hours and the mRNA was extracted for pelleted cells. The steps for cDNA microarray analysis were mainly two: 1 . Total RNA samples were isolated from treated/untreated cells using TRIZOL reagent (Invitrogen); 2. Concentration of purified RNA samples were determined by A260 measurement and the quality was checked by Lab-on-a-chip analysis (total RNA nano biosizing assay, Agilent) with the Agilent 2100 Bioanalyzer RNAs isolated from different tumor tissues, and transcribed in cDNAs, were used to carry out the analysis. The cDNAs from treated $\mathrm{BC}$ were labeled with cy5 red fluorescent dye and untreated $\mathrm{BC}$ with cy3 green fluorescent dye. Hybridization was done on a microarray chip called MWG Human Cancer Array containing 50-mer oligo probes for 1920 genes (1853 human genes associated with cancer, 27 control genes and 40 replicated genes). Spots of fluorescence intensity were read by dual laser scanner (BioDiscovery) and the values were processed with Mavi Pro-2.6.0. (MWG Biotech), by computing background subtraction, normalization to a number of housekeeping genes, and comparison with untreated cancers. In order to select deregulated genes, we considered the cy5/cy3 normalized ratio (NR), calculated for each gene and by taking the ratio of the intensity in cy5 (Ic5) and the normalized intensities in cy3 (nIc3). Then, to reduce variability, all ratio values were transformed in log base 2. For inclusion of highly deregulated genes, we considered as up-regulated or downregulated genes those with $\log 2(N R)>2.0$ and $\log 2(N R)<-2.0$, respectively. 


\section{Functional enrichment and pathways analysis}

The analysis of the list of the significantly deregulated genes is based on the F-Census database [17] (http://210.46.85.180:8080/fcensus/) in order to extract information from highly inconsistent cancer gene data sources including CGC (Cancer Gene Census), OMIM (Online Mendelian Inheritance in Man), AGCOH(Atlas of Genetics and Cytogenetics in Oncology and Haematology), CancerGenes, TSGDB (Tumor Suppressor Gene Database), TGDBs (Tumor Gene Family Databases), two lists (H-list and R-list) that report genes deregulated in cancer samples and identified by mutational screens of cancer genomes using high-throughput techniques, and post-transcriptional regulation predicted by some microRNA (miRNA) target prediction algorithms, including TargetScan, PicTar, DIANA-microT and MirTarget2. For bioinformatics and functional annotation, we used over-representation analysis (ORA), available in ConsensusPathDB [18] (http://cpdb.molgen.mpg.de/), and aimed at identifying the functional categories and biological pathways among the differentially expressed (DE) genes between treated and untreated cell lines in both cancers. ORA allows interactive querying to perform a functional enrichment (FE) from comparison of two lists of genes (deregulated genes versus the universe, i.e. the human genome). The output links the genes to the corresponding annotations, found in databases, i.e. KEGG, Biocarta, Reactome, wikipathways. Among these examples, the ConsensusPathDB latest version - release 26 - was used.

\section{Transcriptional and post-transcriptional regulatory network analysis}

The detection of Transcription Factors (TFs) predicted to regulate the list of significantly deregulated genes upon DAC treatment in BC, was performed using TFactS [19] (http:// www.tfacts.org/). TFactS DB contains genes responsive to TFs, according to experimental evidence reported in literature, and reports two datasets: (i) A sign-sensitive catalogue that indicates the type (up/down) of TF regulation exerted on its targets; (ii) A sign-less catalogue that includes all regulatory interactions contained in sign sensitive and further interactions without the specific type of regulation. TFactS takes as a query the two lists of up- 1 down-regulated genes and compares them with sign-sensitive catalog of manually curated annotated target genes, then returning the lists of activated and inhibited TFs whose annotated target genes show a significant overlap with the query genes.

We then merged the experimentally validated miRNA-target gene DBs: miRTarBase V.3.5 [20] (http://mirtarbase.mbc.nctu.edu.tw/), miRecords V.3 [21] (http://mirecords.biolead.org/), and miR2Disease [22] (http://www.mir2disease.org/), to build a non-redundant dataset of miRNA-target genes regulatory (human) interactions. We used this consensus dataset to predict the miRNA regulators among the list of significantly deregulated genes. With Cytoscape [23] (http://www.cytoscape.org/) we showed the transcriptional and posttranscriptional regulatory networks whose analysis required a tool called "AdvancedNetworkMerged", merging networks by set operations (union, intersection and difference).

\section{Results}

\section{Pathway signatures after DAC treatment}

Overall, we have identified 335 DE genes in BC, and 195 DE genes in melanoma upon DAC treatment of MCF-7 and A375 cell lines. In particular, we found 221 up-regulated genes in BC and 111 up-regulated genes in melanoma based on log2 (NR) > 2, with 29 genes in common. Then, we found 114 and 84 down-regulated genes based on 
$\log 2(\mathrm{NR})<-2$ in $\mathrm{BC}$ and melanoma, respectively, with 50 down-regulated genes in common, and 13 genes down-regulated in melanoma and up-regulated in BC. We integrated the epigenetically modified genes with the F-census database (see Additional File 1 (AF1) Table 1) and the information about their methylation state when available from previous work [24] centered on DNA methylation profiling in MCF-7 (Additional File 1 Table 2). The following examples are listed as top up-regulated genes in BC (Additional File 1 Table 1):

CTAG1B (cancer/testis antigen 1B) - is a member of cancer/testis (CT) antigens found expressed in normal testis and in many cancers [25,26]. We found CTAG2 upregulated in $\mathrm{BC}$, but down-regulated in melanoma. Thanks to the capacity of a subset of these antigens to activate a spontaneous cellular immune responses in cancer patients [27], they are ideal cancer antigen targets for tumor immunotherapy, especially for BC after DAC treatment as with adult T-cell leukemia/lymphoma (ATLL) [28];

RAB30 - is a member of Ras-associated binding proteins (Rabs), which are involved in regulating different steps during exocytosis [29]. In particular, Rab30 is Golgi-specific Rabs, required for the structural integrity of the Golgi apparatus [30], and it was found hypomethylated in MCF7, together with other up-regulated genes (Additional File 1 Table 2);

MAGEA1 and MAGEB2 - are members of CT antigens; in particular, MAGEA1 was found expressed in $10 \%$ of cancer cells in conjunctival melanomas [31], and could be another candidate working in combination with immunotherapy;

FHL2- is a member of the four-and-a-half-LIM-only protein family, found overexpressed in BC cell lines upon DAC [32]; it can inhibit the proliferation and invasive growth of human breast cancer cells by repressing the functional activity of an inhibitor of DNA binding 3 (ID3) [33].

The following examples are listed as top up-regulated genes in melanoma (Additional file 1 Table 1):

S100A2 - is an EF hand calcium binding protein A2, given that the S100 gene family includes at least 13 members that are involved in the regulation of a number of cellular processes such as cell cycle progression and differentiation. It is a potential biomarker in several cancers including melanoma and $\mathrm{BC}$, and may have a tumor suppressor function [34]. Its expression is stimulated by Jun transcription factor which was found up-regulated upon DAC treatment in both cancers;

IGF2 (insulin-like growth factor 2) - is up-regulated in BC. It was recently shown that the epigenetic alterations of IGF-2 are associated with development and progression of hepatocellular carcinoma (HCC) [35];

FANCA (Fanconi anemia, complementation group A) - mutations in this gene represent the most common cause of Fanconi anemia. There are 15 known Fanconi anemia genes involved in different pathways that coordinate multiple DNA repair [36].

The following genes commonly down-regulated in both cancer cell lines after DAC were found:

CDKN2B - is a cyclin-dependent kinase inhibitor 2B (known also as p15, and it inhibits CDK4) and a key regulator of biological processes repressing cell cycle progression by inhibition of cdk4 and cdk6 [37]. This gene is known as a melanoma susceptibility gene and mutations carrier in CDKN2A, correlated with a higher risk of BC. Since down-regulation upon DAC treatment results in both cancer cell lines, it may 
represent a side effect of treatment. The balance between DNA methylation and demethylation is a critical regulator of the methylation status of cyclin-dependent kinase inhibitor [38]. In fact, it is known that the demethylating agents function as DNA methyltransferase inhibitors, can be incorporated into the genome during DNA replication, and bind DNA methyltransferases that have the catalytic domain. This may lead to global hypomethylation and re-expression of both tumor suppressor genes and proto-oncogenes misregulation [39];

CDH6 (cadherin 6) - is a membrane glycoprotein and a member of the cadherin superfamily type II involved in cell-cell adhesion, differentiation and morphogenesis. The aberrant expression of cadherin- 6 correlates with a poor prognosis in patients with E-cadherin-absent Renal cell carcinomas (RCC) and could be a useful tool to estimate the malignancy potential [40];

CDH7 (cadherin 7) - is another member of the cadherin superfamily type II. It was shown that $\mathrm{CDH} 7$ plays a role in tumor development of malignant melanoma cells by interacting with melanoma inhibitory activity protein (MIA) and migration melanoma cell [41].

The top down-regulated genes in the A375 cell line are listed below:

BAI1 (brain-specific angiogenesis inhibitor 1) - is a tumor suppressor gene and transmembrane protein with anti-angiogenic and antiproliferative activity [42];

FKSG2 - is a tumor protein, translationally-controlled 1 pseudogene, which we found down- regulated in $\mathrm{BC}$ too.

We found discrepancy in the regulation of some genes upon DAC treatment, with evidence of genes down-regulated in melanoma and up-regulated in BC. Examples worth of explanation are the following:

1. CFLAR - is a CASP8 and FADD-like apoptosis regulator, and structurally similar to caspase-8. It is found expressed in pulmonary metastases in osteosarcoma patients and human xenografts [43]. Its down-regulation contributes to apoptosis in human lung cancer cells, suggesting that its targeting may represent a promising therapeutic strategy for the cancer patients with over-expression of this gene [44];

2. MDM2 (Mouse double minute 2) - is an oncogene E3 ubiquitin protein ligase, regulated by p53 in case of DNA damage and part of an autoregulatory negative feedback loop [45];

3. PRKCH (protein kinase $\mathrm{C}$, eta) - is a calcium-independent and phospholipidsdependent protein kinase. It is mostly expressed in epithelial tissues and has been shown to activate the protein kinase cascade that targets CCAAT/enhancer-binding protein alpha (CEBPA), following the same expression as $\mathrm{PRKCH}$ in both cancer cell lines [46].

Using ORA from the consensusPathDB, we found a list of over-represented functional categories and pathways referring to up- and down-regulated genes in the cancer cell lines. The enriched pathways are reported in Additional File 1 Table 3. The graphical tool in consensusPathDB was used to select the most interesting pathways involved in up- and down-regulated genes (Figure 1and Figure 2). The node size indicates the size of the gene set; the node color corresponds to the p-value (deeper red means smaller p-value); the edge color represents the number of shared genes in the predefined dataset; 


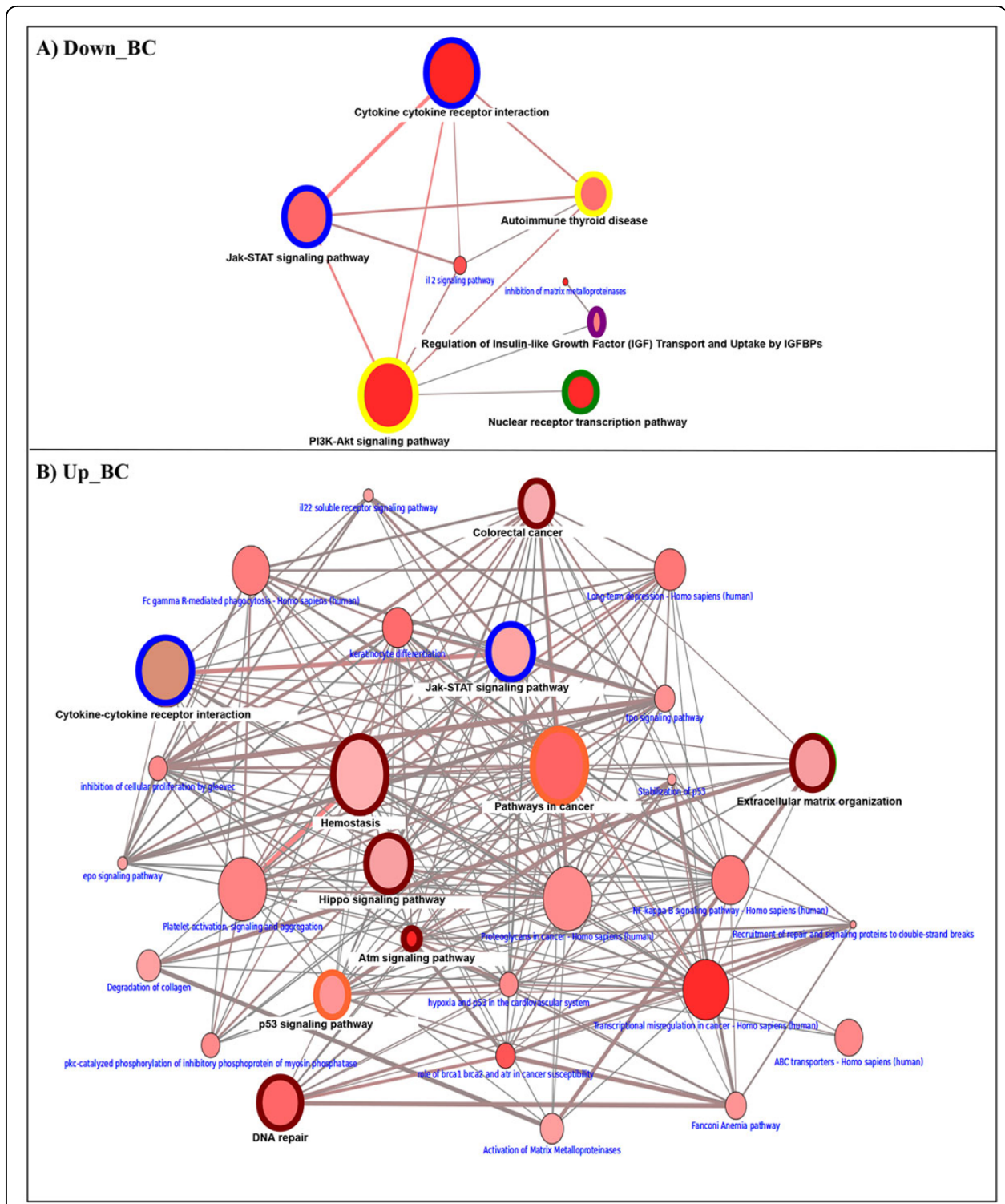

Figure 1 Breast cancer and integrated Melanoma pathway landscape. The color node border indicates the common pathways among differentially expressed genes in two cancer cell lines: Green for downregulated in both tumors; Dark red for up-regulated genes in both tumors; Blue for up/down-regulated in $\mathrm{BC}$ and down in melanoma; Yellow for down-regulated in BC and up/down-regulated genes in melanoma; Orange for up-regulated and down-regulated genes in $B C$ and melanoma, respectively and pink color for down-regulated and up-regulated genes in melanoma and BC, respectively. A) Enriched pathways among down- regulated genes in $\mathrm{BC}$; $\mathrm{B}$ ) Enriched pathways among up-regulated genes in $\mathrm{BC}$.

the color node border indicates the common pathways among genetically regulated genes. In Figure 1 and Figure 2 we show the common pathways regulated upon DAC treatment; in Table 1 we report the common enriched pathways among up-regulated genes.

The commonly enriched pathways associated to up-regulated genes is ATM signaling (Ataxia telangiectasia mutated) which is associated with the activation of p73, a member of the p53 tumor suppressor family regulating cell cycle and apoptosis after DNA damage, and found inactivated in many tumor histotypes $[47,48]$. The 


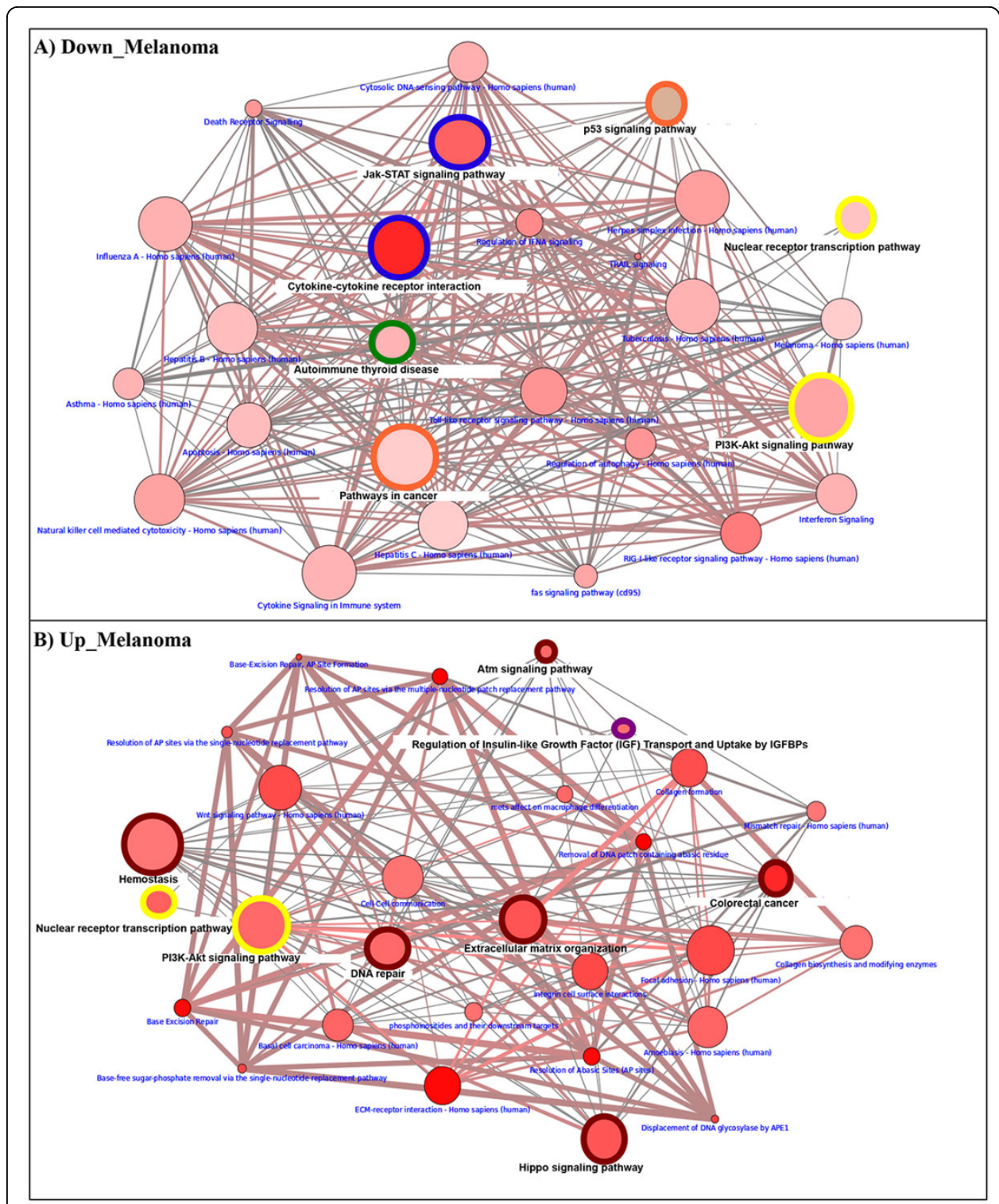

Figure 2 Melanoma and integrated Breast cancer pathway landscape. The color node border indicates the common pathways among differentially expressed genes in two cancer cell lines: Green for downregulated in both tumors; Dark red for up-regulated genes in both tumors; Blue for up/down-regulated in $\mathrm{BC}$ and down in melanoma; Yellow for down-regulated in BC and up/down-regulated genes in melanoma; Orange for up-regulated and down-regulated genes in $B C$ and melanoma, respectively and pink color for down-regulated and up-regulated genes in melanoma and BC, respectively. A) Enriched pathways among down- regulated genes in melanoma. B) Enriched pathways among up-regulated genes in melanoma.

methylation status of p73 was shown to be common in patients with MDS and associated with poor prognosis [49]. p73 plays also a key role in p53 signaling pathway exerting an anticarcinogenic effect [50]; the activation of this signaling pathway induced by a natural diterpenoid compound was associated to G(2)/M cell cycle arrest in human melanoma cells to repair DNA damage. Similarly, such mechanism could be activated here, due to the up-regulation of genes involved in DNA repair (e.g. ATM is an important cell cycle checkpoint kinase). Then, evidence includes XPC that plays a central role in the recognition of DNA damage; LIG3 which is involved in excision 
Table 1 The common pathways associated to up-regulated genes.

\begin{tabular}{|c|c|c|c|c|}
\hline Type CCL & Pathway name & Genes & p-value & q-value \\
\hline $\mathrm{BC}$ & Atm signaling & ATM; MDM2; RBBP8; JUN; TP73 & $2.81 \mathrm{E}-06$ & 7.96E-04 \\
\hline Melanoma & & JUN; TP73 & $5.73 \mathrm{E}-03$ & $3.42 \mathrm{E}-02$ \\
\hline$B C$ & Colorectal cancer & JUN; TGFB3; RAF1; DCC & $9.04 \mathrm{E}-03$ & $6.89 \mathrm{E}-02$ \\
\hline Melanoma & & MSH2; JUN; DCC; TCF7L2 & $6.36 \mathrm{E}-04$ & 1.45E-02 \\
\hline BC & DNA repair & XPC; LIG3; ATM; TDG; ERCC6; FANCE; FANCC; RAD50 & $1.30 \mathrm{E}-04$ & 9.17E-03 \\
\hline Melanoma & & MUTYH; POLD1; TDG; FANCA & $5.94 \mathrm{E}-03$ & $3.42 \mathrm{E}-02$ \\
\hline$B C$ & $\begin{array}{l}\text { Extracellular } \\
\text { matrix } \\
\text { organization }\end{array}$ & MMP14; TGFB3; ADAM17; CTSG; COL1A2; MMP8; CTSL2 & 4.01E-03 & $5.02 \mathrm{E}-02$ \\
\hline Melanoma & & COL3A1; COL1A2; ITGB4; TIMP1; COL1A1 & $2.78 \mathrm{E}-03$ & 2.38E-02 \\
\hline$B C$ & Hemostasis & $\begin{array}{l}\text { IGF2; PRKCH; PPP2R1B; TGFB3; AKAP1; SERPINC1; VAV2; } \\
\text { PRKCE; ANGPT2; GNA12; GNA13; ABCC4; RAF1 }\end{array}$ & $9.01 \mathrm{E}-03$ & $6.89 \mathrm{E}-02$ \\
\hline Melanoma & & IGF2; TIMP1; SERPINC1; CD47; PRKCE; GRB7; PDPK1; ITPR3 & $9.42 \mathrm{E}-03$ & 4.30E-02 \\
\hline BC & Hippo signaling & WNT2B; PPP2R1B; TGFB3; WNT1; ID2; TP73; TEAD4 & 4.62E-03 & 5.02E-02 \\
\hline Melanoma & & DVL3; LATS1; FZD4; TP73; TCF7L2 & $3.11 \mathrm{E}-03$ & $2.50 \mathrm{E}-02$ \\
\hline
\end{tabular}

repair; TDG which is important in DNA demethylation via the base excision repair pathway [51], in cellular defense against genetic mutation, in regulation of the epigenome and gene expression in non-melanoma skin cancer [52]; MUTYH that is involved in oxidative DNA damage repair in mammalian cells [53]. Another interesting pathways activated in both cancer cell lines is the extracellular matrix organization which involves up-regulation of different collagen genes like (COL1A1, COL1A2, COL3A1), and matrix metallopeptidase like (MMP8, MMP14).

Table 2 lists the common pathways among down-regulated genes, and also the genes involved in each specific pathway. We found also Nuclear receptor transcription pathway enriched among down-regulated genes like NR1D1, which is a member of the nuclear receptor subfamily 1 , also found expressed in human endometrial stromal and epithelial cells [54].

Table 2 The common pathways associated to down-regulated genes.

\begin{tabular}{|c|c|c|c|c|}
\hline Type CCL & Pathway name & Genes & p-value & q-value \\
\hline Melanoma & $\begin{array}{l}\text { Cytokine-cytokine } \\
\text { receptor } \\
\text { interaction* }\end{array}$ & $\begin{array}{l}\text { CSF2RB; IL2RA; TNFSF18; TNFSF15; IFNA1; IL13; IL4; } \\
\text { IL12A; IFNB1; TNFRSF10B; BMPR1B; IFNA8; IFNA5; } \\
\text { TNFRSF18; LIFR }\end{array}$ & $3.63 \mathrm{E}-11$ & 4.24E-09 \\
\hline $\mathrm{BC}$ & & $\begin{array}{l}\text { IL2RA; IFNA8; IFNA1; BMPR1B; TNFSF8; IL2; IL26; } \\
\text { TNFRSF18 }\end{array}$ & $2.92 \mathrm{E}-04$ & 1.61E-02 \\
\hline Melanoma & $\begin{array}{l}\text { Jak-stat signaling } \\
\text { pathway* }\end{array}$ & $\begin{array}{l}\text { CSF2RB; IL2RA; IFNA8; IFNA1; IL13; IFNA5; IFNB1; IL4; } \\
\text { IL12A; LIFR }\end{array}$ & $3.09 \mathrm{E}-08$ & $1.81 \mathrm{E}-06$ \\
\hline $\mathrm{BC}$ & & IL2RA; IFNA1; IFNA8; IL2; IL26 & $3.41 \mathrm{E}-03$ & $6.86 \mathrm{E}-02$ \\
\hline Melanoma & $\begin{array}{l}\text { PI3K-AKT signaling } \\
\text { pathway\# }\end{array}$ & $\begin{array}{l}\text { IL2RA; IFNA8; IFNA1; IFNA5; IFNB1; IL4; FGF12; MDM2; } \\
\text { FGF20 }\end{array}$ & $2.11 \mathrm{E}-04$ & $1.76 \mathrm{E}-03$ \\
\hline BC & & $\begin{array}{l}\text { IL2RA; IFNA8; IFNA1; THBS2; RXRA; IL2; FGF12; TSC2; } \\
\text { FGF20 }\end{array}$ & 3.39E-04 & 1.61E-02 \\
\hline Melanoma & $\begin{array}{l}\text { Nuclear receptor } \\
\text { transcription } \\
\text { pathway\# }\end{array}$ & NR1D1; AR; NR2E1 & 3.84E-03 & 1.36E-02 \\
\hline$B C$ & & NR1D1; NR2E1; RXRA; RORB & $3.43 \mathrm{E}-04$ & $1.61 \mathrm{E}-02$ \\
\hline Melanoma & $\begin{array}{l}\text { Autoimmune } \\
\text { thyroid disease }\end{array}$ & IL4; IFNA8; IFNA1; IFNA5 & 2.71E-04 & $1.98 \mathrm{E}-03$ \\
\hline $\mathrm{BC}$ & & IFNA1; IFNA8; IL2 & 4.56E-03 & 7.15E-02 \\
\hline
\end{tabular}

Some of the listed pathways are associated to up-regulated genes in BC (*) and in melanoma (\#). 
In Table 3, we reported pathways that we found commonly enriched among upregulated genes in one cancer cell line and down-regulated genes in the other cell line. The first example is the p53 signaling pathway, associated to up-regulated genes in MCF7. This is the case of PPM1D, a protein phosphatase, Mg2+/Mn2+ dependent, 1D, found over-expressed in different types of human tumors with poor prognosis, and negatively regulating p38 MAPK activity to reduce the phosphorylation of p53 [55,56]. PPM1D could dephosphorylate ATM and MDM2 [57] involved in this pathway. Another key regulator in this pathway is TNFRSF10B (tumor necrosis factor receptor superfamily, member 10b), involved in apoptosis transduction [58]. Then, regulation of insulin-like growth factor (IGF) transport and uptake by IGFBPs that involves up-regulated genes in A375, e.g. IGF2 (insulin-like growth factor 2, also called somatomedin A), implicated in growth and development. It is known that epigenetic alterations of IGF2 is correlated with severity of hepatocellular carcinoma development and progression. Additionally, down-regulated genes are involved, e.g. MMP2, which is a metalloproteinase implicated in cancer progression and metastasis whose increase in expression is associated with a poor prognosis $[59,60]$.

\section{Transcriptional and post-transcriptional regulatory networks}

We reported in Additional File 1 Table 4 the TFs that were found significantly enriched among the deregulated genes by using the TFactS analysis tool. We built with "AdvancedNetworkMerged" in Cytoscape the union of transcriptional network and the miRNA regulatory network to show TFs and miRNAs experimentally validated as candidate regulators of the epigenetically modified genes in BC (Figure 3) and melanoma (Figure 4). Then, we extracted the sub-networks resulting from the intersection of the two regulatory networks (Figure 5). As a result, the epigenetic profile is described in an integrative network context.

In Figure 3, we reported the regulatory map of DE genes in MCF7. This network is composed by 300 edges, 242 nodes that correspond to 66 up-regulated and 28 downregulated genes, 67 predicted TFs and 85 miRNAs. Among the predicted TFs, we found four - CEPBA, JUN, RUNX2 and ID2- that are up-regulated upon DAC treatment. In Figure 4, we reported the regulatory map of the epigenetically modified genes in A375 which is composed by 218 edges, 173 nodes matching 28 up-regulated and 29

Table 3 The rest of common pathways associated to up or down-regulated genes in both cancer cell lines.

\begin{tabular}{|c|c|c|c|c|}
\hline $\begin{array}{l}\text { Gene } \\
\text { expression }\end{array}$ & Pathway name & Genes & p-value & q-value \\
\hline Up-BC & P53 signaling pathway & ATM; MDM2; PPM1D; APAF1; TP73 & $2.01 \mathrm{E}-03$ & $4.56 \mathrm{E}-02$ \\
\hline \multirow{2}{*}{$\begin{array}{l}\text { Down- } \\
\text { melanoma }\end{array}$} & & TNFRSF10B; MDM2; CASP8; BAI1 & 7.57E-04 & 4.12E-02 \\
\hline & Pathways in cancer & $\begin{array}{l}\text { CDKN2B; CEBPA; CASP8; AR; FGF12; } \\
\text { MDM2; FGF20 }\end{array}$ & 3.65E-03 & $1.33 \mathrm{E}-02$ \\
\hline Up-BC & & $\begin{array}{l}\text { PGF; PTGS2; CEBPA; TGFB3; WNT1; JUN; } \\
\text { DCC; STAT5B; CSF2RA; WNT2B; MDM2; } \\
\text { RAF1; PAX8; IL8 }\end{array}$ & $1.05 \mathrm{E}-04$ & 9.17E-03 \\
\hline $\begin{array}{l}\text { Up- } \\
\text { melanoma }\end{array}$ & $\begin{array}{l}\text { Regulation of insulin-like growth } \\
\text { factor (IGF) transport and uptake } \\
\text { by IGFBPs }\end{array}$ & IGF2; IGFBP6 & 7.77E-03 & 4.09E-02 \\
\hline Down-BC & & IGFBP1; MMP2 & 8.00E-03 & 9.59E-02 \\
\hline
\end{tabular}




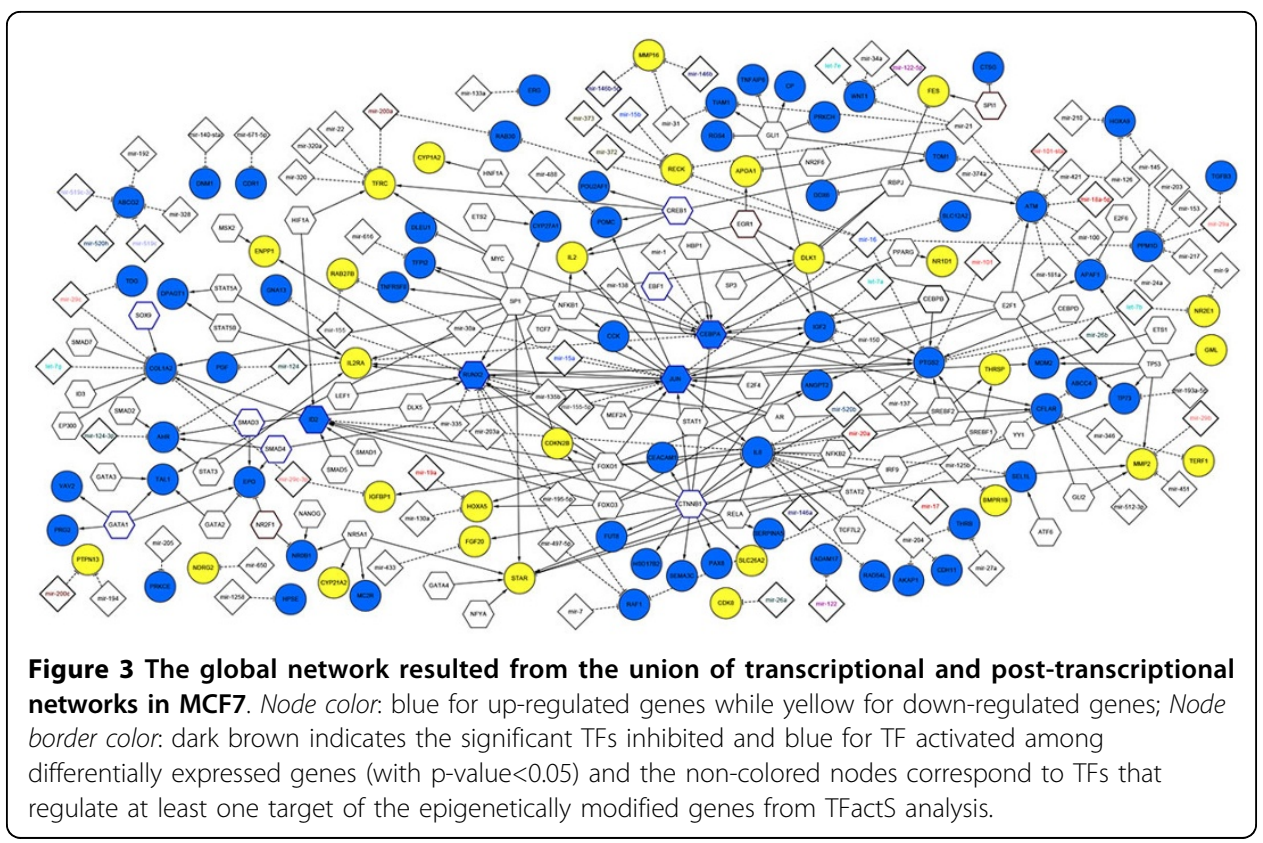

down-regulated genes, 53 predicted TFs and 66 miRNAs. Among the predicted TFs we found three - CEPBA, JUN and AR- that are deregulated upon DAC treatment. In Figure 5, we reported the sub-network resulted from the intersection of the two regulatory networks in MCF7 and A375. This network is composed by 117 edges; 94 nodes that correspond to 12 up-regulated, and 11 to down-regulated genes in both cells, while 4 are up-regulated in BC and down-regulated in melanoma. Then, there are 41 predicted TFs and 28 miRNAs.

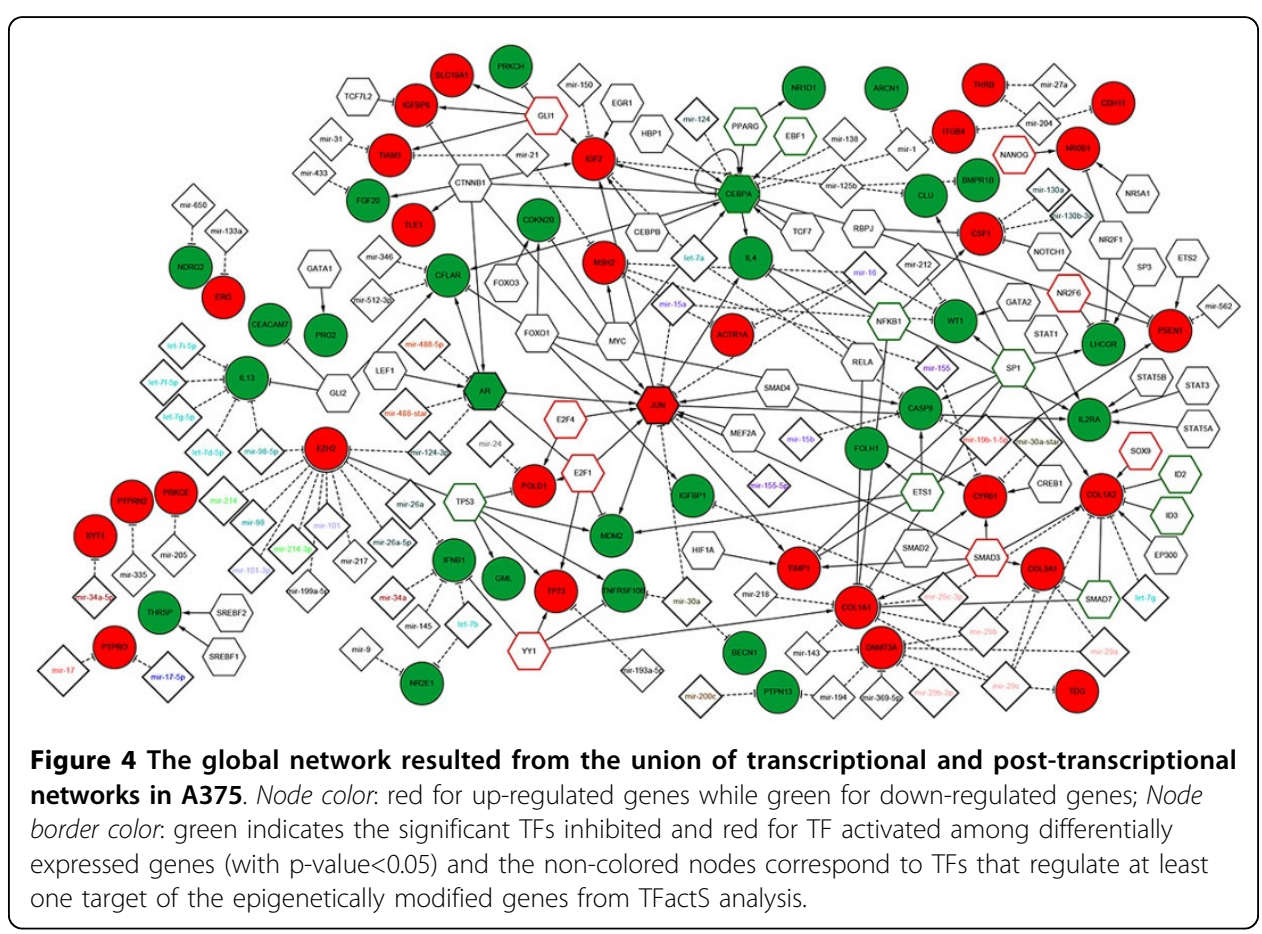




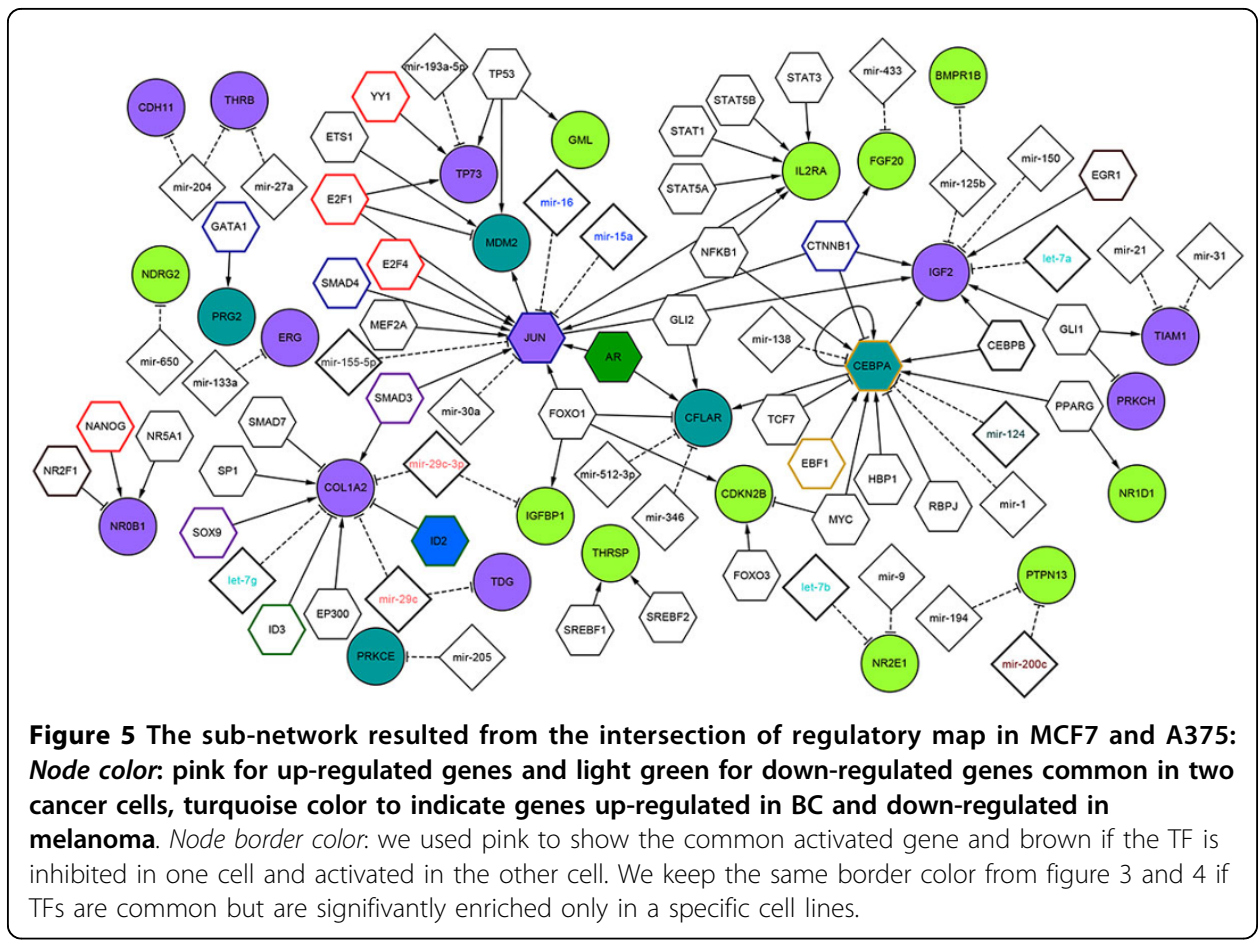

We found that the common up-regulated TF in both cell lines is JUN, a protoncogene that plays a dual role in regulation of tumor cell proliferation [61], and recently reported as candidate biomarker for personalized therapy in thymic epithelial tumors [62]. Its up-regulation is associated to the activation of IGF2 in both cell lines, and to an increased expression of MDM2 in MCF7. JUN represents a target of different miRNAs. For instance, miRNAs-15a/16 cluster, considered a tumor suppressor found deleted in different malignancies [63,64], or miR-155 reported as repressor of JUN in human dermal fibroblasts in vitro [65]. Interestingly, the up-regulation of Jun TF in MCF7 is connected to the activation of TIMP1, a mettallopeptidase inhibitor whose overexpression contributes to antimetastatic effect in $\mathrm{BC}$ [66], and to the activation of NQO1 (NADPH quinone oxidoreductase 1), playing a cytoprotective role and found associated to increase cell-sensitivity to $\mathrm{BC}$ anticancer treatment [67].

An important JUN target is the SERPINB5 (serpin peptidase inhibitor clade B, member 5), found also up-regulated upon DAC treatment in MCF7, and known as an important suppressor of the invasion and migration of cancer cells [68]. Then, another target is STAT5B (signal transducer and activator of transcription 5B), a member of the STAT family of TFs, normally activated in response to cytokines and growth factors signals. It has been shown that the regulation of STAT1/STAT5 signalling pathway mediated by STAT5B is important in the regulation of essential functions in the mammary gland [69]. The TFact tool reported among the up-regulated target of STAT5B the DPAGT1 target, which is an enzyme that catalyzes glycoprotein biosynthesis and was found to be targeted also by wnt $/ \beta$-Catenin signalling pathways, mediating a variety of critical developmental processes [70].

Another important key regulator is CEBPA, which is down-regulated in melanoma and up-regulated in $\mathrm{BC}$, a key TF involved in the regulation of cellular processes, 
especially in Hematopoietic system [71]. It was found epigenetically modified and posttranscriptionally regulated by miR-124 after DAC and trichostatin treatment in AML [72]. The repression of CEBPA by miR-138 was found to inhibit adipogenic differentiation of human adipose tissue-derived mesenchymal stem cells [73]. The post-transcriptional mechanism of regulation may be the same also in both cancer cell lines (Figure 4). CEPBA, being up-regulated in MCF7, cooperates with RUNX2 (runt-related TF 2) to activate PTGS2 (prostaglandin-endoperoxide synthase 2). The latter is known as cyclooxygenase-2 (COX-2), an inducible enzyme responsible in the prostanoid biosynthesis and involved in inflammation and mitogenesis [74]. PTGS2 is a target for development of anticancer therapy, and the use of anti-inflammatory PTGS2 inhibitors represents a promising strategy in the treatment of solid tumors [75]. In the A375 cell line, the down-regulation of CEPBA could be responsible of the up-regulation of PSEN1 (presenilin 1), mutated in patient with sporadic early-onset Alzheimer's disease [76].

An important up-regulated gene in the A375 cell line is EZH2 (Enhancer of zeste homolog 2), a member of polycomb protein and a part of polycomb repressive complex, implicated in cancer stem cell maintenance and metastasis in breast and pancreatic cancer $[77,78]$, and it could be considered as a prognostic marker in renal cell carcinoma [79]. EZH2 is repressed by several miRNAs. It was reported in [80] that the loss of miR-101 expression correlates with the increase in EZH2 expression in invasive squamous cell carcinoma.

\section{Discussion}

An accurate curation of pathway database with and regulatory relations was our preliminary step to draw a representative map of the major gene regulators and pathway landscapes involved in the DAC treatment of the MCF7 breast and the A375 melanoma cell lines. Then, a dissection of the key pathways and the knowledge of the interconnectivity between its components have been computed because conceived as valuable knowledge to gain towards new therapeutic approaches targeting the common genes involved in oncogenic pathways activated upon DAC treatment.

Our results are in agreement with previous studies showing the mechanism underlying the anticancer effect of DAC treatment, and its involvement in activation of the ATM signaling pathways in cancer cell lines [81]. The identification of a potential TF-miRNA regulatory network contributes to shed light on the potential molecular mechanism played by DAC treatment in both cancer cells. It is important to notice that DAC could have a different effect in the regulation of key regulators depending on the cancer cell line. One of the controversial molecular mechanisms is the increase of CEBPA expression in MCF7 and its down-regulation in A375. However, its posttranscriptional regulation by miR-124 results affected by demethylating agent, suggesting that the effectiveness of epigenetic therapy already shown in AML could be further investigated in both the cancer cell lines examines here.

Notably, some other interesting aspects are worth deeper discussion. The DAC treatment in MCF-7 and A375 cells induces down-regulation of different cytokines and interferons genes that represent key regulators of the cytokine-cytokine receptor interaction, Jak-STAT and PI3K-AKT signaling pathway, and they are associated to autoimmune thyroid disease. IL2RA and IL-2 are the common genes among all these pathways. The up-regulation of the interleukin-2 (IL-2) and its receptor alpha (IL2RA) 
are associated with the malignancy of the infiltrating human breast cancer. It may be the case that their down-regulation could produce an anticancer effect. The deregulation of these cytokines and interferons genes may suggest a potential role as breast cancer biomarker, or their possible use for novel DAC-combined treatments.

All these evidences might help the design of new therapeutic formulations, and the identification of the best therapeutic combinations between the use of demethylating agent as DAC and immonotherapy, e.g directly targeting the CT antigens, the cancer antigens, or other therapeutic tools that could improve the anticancer effect of the demethylating agent, obtaining therefore more efficient BC therapy. Moreover, we are confident that gene expression and pathway signatures could give aid to the discovery of relevant connections among molecular mechanisms and drugs so as to significantly contribute to the improvement of drug for cancer therapy. As a follow up agenda for future research, we are planning to extend our analysis to other tumor histotypes, and also to use novel gene expression profiling (exploiting transcriptome landscapes from RNA-Seq) for refinement of pathway signatures.

\section{Additional material}

Additional File 1: AF1 Table 1: The list with the expression values of deregulated genes in both cancer cell lines and the corresponding information extracted from F-census about Gene type: oncogene or TSG (tumor suppressor gene) extracted from both CGC and TSGDB; cancer type according to the classes provided in COSMIC; mutation frequency calculated by six high-throughput mutational screen data of cancer genomes; the number of miRNA predicted to regulate the target cancer genes, extracted from prediction tools including TargetScans, PicTar, DIANA-microT and MirTarget2. AF1 Table 2: Additional information for deregulated genes in MCF7 provided from detection of DNA methylation state in BC. AF1 Table 3: Enriched Gene Ontology Biological processes (GO BP) associated to discordant DE genes. AF1 Table 4: The list of the most enriched functional categories and pathways among the epigenetically modified genes in both cell lines extracted from ConsensusPathDB using ORA tool. AF1 Table 5: The list of TFs found significantly enriched among the epigenetically modified genes in BC and

Melanoma. AF1 Figure 1: Graphical representation of the most enriched GO BP among discordant DE genes.

Competing interests

The authors declare that they have no competing interests.

\section{Authors' contributions}

ME participated in design of the study, performed the bioinformatic analysis and wrote the paper. DL carried out the microarray experiments. CC design and coordination of the study. EC design and coordination of the study and wrote the paper. All authors read and approved the final manuscript.

\section{Acknowledgements}

The authors thanks the IWBBIO 2013 Special Issues' Editors. ME acknowledges partial support from the Flagship project InterOmics (PB.P05), funded by the Italian MIUR and CNR organizations.

\section{Declarations}

Publication funding for this article has come from CNR, Pisa.

This article has been published as part of Theoretical Biology and Medical Modelling Volume 11 Supplement 1, 2014: Selected articles from the 1st International Work-Conference on Bioinformatics and Biomedical Engineering-IWBBIO 2013. The full contents of the supplement are available online at http://www.tbiomed.com/supplements/11/S1.

\footnotetext{
Authors' details

'Laboratory of Integrative Systems Medicine (LISM), Institute of Clinical Physiology (IFC) \& Institute of Informatics and Telematics (IIT), National Research Council (CNR), Pisa, 56124, Italy. ${ }^{2}$ Department of Experimental Biomedicine and Clinical Neuroscience, Policlinico Universitario, Palermo, 90127, Italy. ${ }^{3}$ Experimental Oncology Unit, Institute of Clinical Physiology (IFC), National Research Council (CNR), Siena, 53100, Italy. ${ }^{4}$ Center for Computational Science, Miller School of Medicine, University of Miami, Miami, Florida 33136, USA.
}

Published: 7 May 2014

References

1. Sharma S, Kelly TK, Jones PA: Epigenetics in cancer. Carcinogenesis 2010, 31:27-36. 
2. Boumber Y, Issa J-PJ: Epigenetics in cancer: what's the future? Oncol Williston Park N 2011, 25:220-226, 228.

3. Yoo CB, Jones PA: Epigenetic therapy of cancer: past, present and future. Nat Rev Drug Discov 2006, 5:37-50.

4. Grønbaek K, Hother C, Jones PA: Epigenetic changes in cancer. Apmis Acta Pathol Microbiol Immunol Scand 2007, 115:1039-1059

5. Momparler RL: Epigenetic therapy of cancer with 5-aza-2'-deoxycytidine (decitabine). Semin Oncol 2005, 32:443-451.

6. Lemaire M, Momparler LF, Bernstein ML, Marquez VE, Momparler RL: Enhancement of antineoplastic action of 5-aza2'-deoxycytidine by zebularine on L1210 leukemia. Anticancer Drugs 2005, 16:301-308.

7. Momparler RL, Bovenzi V: DNA methylation and cancer. J Cell Physiol 2000, 183:145-154.

8. Fang F, Balch C, Schilder J, Breen T, Zhang S, Shen C, Li L, Kulesavage C, Snyder AJ, Nephew KP, Matei DE: A phase 1 and pharmacodynamic study of decitabine in combination with carboplatin in patients with recurrent, platinumresistant, epithelial ovarian cancer. Cancer 2010, 116:4043-4053.

9. Kim JH, Kang S, Kim TW, Yin L, Liu R, Kim SJ: Expression profiling after induction of demethylation in MCF-7 breast cancer cells identifies involvement of TNF-a mediated cancer pathways. Mol Cells 2012, 33:127-133.

10. Tawbi HA, Beumer JH, Tarhini AA, Moschos S, Buch SC, Egorin MJ, Lin Y, Christner S, Kirkwood JM: Safety and efficacy of decitabine in combination with temozolomide in metastatic melanoma: a phase I/II study and pharmacokinetic analysis. Ann Oncol Off J Eur Soc Med Oncol Esmo 2013, 24:1112-1119.

11. Borg A, Sandberg T, Nilsson K, Johannsson O, Klinker M, Måsbäck A, Westerdahl J, Olsson H, Ingvar C: High frequency of multiple melanomas and breast and pancreas carcinomas in CDKN2A mutation-positive melanoma families. J Natl Cancer Inst 2000, 92:1260-1266.

12. Debniak T, Scott RJ, Huzarski T, Byrski T, Masojć B, van de Wetering T, Serrano-Fernandez P, Górski B, Cybulski C, Gronwald J, Debniak B, Maleszka R, Kładny J, Bieniek A, Nagay L, Haus O, Grzybowska E, Wandzel P, Niepsuj S, Narod SA, Lubinski J: XPD common variants and their association with melanoma and breast cancer risk. Breast Cancer Res Treat 2006, 98:209-215.

13. Debniak T, Scott R, Masojc B, Serrano-Fernández P, Huzarski T, Byrski T, Debniak B, Górski B, Cybulski C, Medrek K, Kurzawski G, van de Wetering T, Maleszka R, Kładny J, Lubinski J: MC1R common variants, CDKN2A and their association with melanoma and breast cancer risk. Int J Cancer J Int Cancer 2006, 119:2597-2602.

14. Goggins W, Gao W, Tsao H: Association between female breast cancer and cutaneous melanoma. Int J Cancer J Int Cancer 2004, 111:792-794.

15. Huber C, Bouchardy C, Schaffar R, Neyroud-Caspar I, Vlastos G, Le Gal F-A, Rapiti E, Benhamou S: Antiestrogen therapy for breast cancer modifies the risk of subsequent cutaneous melanoma. Cancer Prev Res Phila Pa 2012, 5:82-88.

16. La Porta CAM, Zapperi S: Human breast and melanoma cancer stem cells biomarkers. Cancer Lett 2012.

17. Gong X, Wu R, Zhang Y, Zhao W, Cheng L, Gu Y, Zhang L, Wang J, Zhu J, Guo Z: Extracting consistent knowledge from highly inconsistent cancer gene data sources. BMC Bioinformatics 2010, 11:76.

18. Kamburov A, Stelzl U, Lehrach H, Herwig R: The ConsensusPathDB interaction database: 2013 update. Nucleic Acids Res 2013, 41(Database):D793-800.

19. Essaghir A, Toffalini F, Knoops L, Kallin A, van Helden J, Demoulin J-B: Transcription factor regulation can be accurately predicted from the presence of target gene signatures in microarray gene expression data. Nucleic Acids Res 2010, 38:e120.

20. Hsu S-D, Lin F-M, Wu W-Y, Liang C, Huang W-C, Chan W-L, Tsai W-T, Chen G-Z, Lee C-J, Chiu C-M, Chien C-H, Wu M-C, Huang C-Y, Tsou A-P, Huang H-D: miRTarBase: a database curates experimentally validated microRNA-target interactions. Nucleic Acids Res 2011, 39(Database):D163-169.

21. Xiao F, Zuo Z, Cai G, Kang S, Gao X, Li T: miRecords: an integrated resource for microRNA-target interactions. Nucleic Acids Res 2009, 37(Database):D105-110.

22. Jiang $Q$, Wang $Y$, Hao Y, Juan L, Teng M, Zhang X, Li M, Wang G, Liu Y: miR2Disease: a manually curated database for microRNA deregulation in human disease. Nucleic Acids Res 2009, 37(Database):D98-104.

23. Smoot ME, Ono K, Ruscheinski J, Wang P-L, Ideker T: Cytoscape 2.8: new features for data integration and network visualization. Bioinforma Oxf Engl 2011, 27:431-432.

24. Li J, Gao F, Li N, Li S, Yin G, Tian G, Jia S, Wang K, Zhang X, Yang H, Nielsen AL, Bolund L: An improved method for genome wide DNA methylation profiling correlated to transcription and genomic instability in two breast cancer cell lines. Bmc Genomics 2009, 10:223.

25. Caballero OL, Chen Y-T: Cancer/testis (CT) antigens: potential targets for immunotherapy. Cancer Sci 2009, 100:2014-2021.

26. Simpson AJG, Caballero OL, Jungbluth A, Chen Y-T, Old L: Cancer/testis antigens, gametogenesis and cancer. Nat Rev Cancer 2005, 5:615-625.

27. Gnjatic $S$, Nishikawa $H$, Jungbluth $A A$, Güre $A O$, Ritter $G$, Jäger $E$, Knuth $A$, Chen Y-T, Old LJ: NY-ESO-1: review of an immunogenic tumor antigen. Adv Cancer Res 2006, 95:1-30.

28. Nishikawa H, Maeda Y, Ishida T, Gnjatic S, Sato E, Mori F, Sugiyama D, Ito A, Fukumori Y, Utsunomiya A, Inagaki H, Old L, Ueda R, Sakaguchi S: Cancer/testis antigens are novel targets of immunotherapy for adult T-cell leukemia/ lymphoma. Blood 2012, 119:3097-3104.

29. Hutagalung AH, Novick PJ: Role of Rab GTPases in membrane traffic and cell physiology. Physiol Rev 2011, 91:119-149.

30. Kelly EE, Giordano F, Horgan CP, Jollivet F, Raposo G, McCaffrey MW: Rab30 is required for the morphological integrity of the Golgi apparatus. Biol Cell Auspices Eur Cell Biol Organ 2012, 104:84-101.

31. Errington JA, Conway RM, Walsh-Conway N, Browning J, Freyer C, Cebon J, Madigan MC: Expression of cancer-testis antigens (MAGE-A1, MAGE-A3/6, MAGE-A4, MAGE-C1 and NY-ESO-1) in primary human uveal and conjunctival melanoma. Br J Ophthalmol 2012, 96:451-458.

32. Putnik $M$, Zhao C, Gustafsson J- $\AA$, Dahlman-Wright $K$ : Global identification of genes regulated by estrogen signaling and demethylation in MCF-7 breast cancer cells. Biochem Biophys Res Commun 2012, 426:26-32.

33. Chen $Y-H$, Wu Z-Q, Zhao Y-L, Si Y-L, Guo M-Z, Han W-D: FHL2 inhibits the Id3-promoted proliferation and invasive growth of human MCF-7 breast cancer cells. Chin Med J (Engl) 2012, 125:2329-2333.

34. Naz S, Ranganathan P, Bodapati P, Shastry AH, Mishra LN, Kondaiah P: Regulation of S100A2 expression by TGF- $\beta$ induced MEK/ERK signalling and its role in cell migration/invasion. Biochem J 2012, 447:81-91. 
35. Dong Z, Yao D, Wu W, Qiu L, Yao N, Yan X, Yu D, Chen J: Correlation between epigenetic alterations in the insulin growth factor-Il gene and hepatocellular carcinoma. Zhonghua Gan Zang Bing Za Zhi Zhonghua Ganzangbing Zazhi Chin J Hepatol 2012, 20:593-597.

36. Kim H, Yang K, Dejsuphong D, D'Andrea AD: Regulation of Rev1 by the Fanconi anemia core complex. Nat Struct Mol Biol 2012, 19:164-170

37. Kim WY, Sharpless NE: The regulation of INK4/ARF in cancer and aging. Cell 2006, 127:265-275.

38. Thillainadesan G, Chitilian JM, Isovic M, Ablack JNG, Mymryk JS, Tini M, Torchia J: TGF- $\beta$-dependent active demethylation and expression of the p15ink4b tumor suppressor are impaired by the ZNF217/CoREST complex. Mol Cell 2012, 46:636-649.

39. Haaf T: The effects of 5-azacytidine and 5-azadeoxycytidine on chromosome structure and function: implications for methylation-associated cellular processes. Pharmacol Ther 1995, 65:19-46.

40. Shimazui T, Kojima T, Onozawa M, Suzuki M, Asano T, Akaza H: Expression profile of N-cadherin differs from other classical cadherins as a prognostic marker in renal cell carcinoma. Oncol Rep 2006, 15:1181-1184.

41. Winklmeier A, Contreras-Shannon V, Arndt S, Melle C, Bosserhoff A-K: Cadherin-7 interacts with melanoma inhibitory activity protein and negatively modulates melanoma cell migration. Cancer Sci 2009, 100:261-268.

42. Koh JT, Kook H, Kee HJ, Seo Y-W, Jeong BC, Lee JH, Kim M-Y, Yoon KC, Jung S, Kim KK: Extracellular fragment of brain-specific angiogenesis inhibitor 1 suppresses endothelial cell proliferation by blocking alphavbeta5 integrin. Exp Cell Res 2004, 294:172-184.

43. Rao-Bindal K, Rao CK, Yu L, Kleinerman ES: Expression of c-FLIP in pulmonary metastases in osteosarcoma patients and human xenografts. Pediatr Blood Cancer 2013, 60:575-579.

44. Wang Q, Sun W, Hao X, Li T, Su L, Liu X: Down-regulation of cellular FLICE-inhibitory protein (Long Form) contributes to apoptosis induced by Hsp90 inhibition in human lung cancer cells. Cancer Cell Int 2012, 12:54.

45. Tang J, Agrawal T, Cheng Q, Qu L, Brewer MD, Chen J, Yang X: Phosphorylation of Daxx by ATM contributes to DNA damage-induced p53 activation. Plos One 2013, 8:e55813.

46. Artemenko Y, Gagnon A, Aubin D, Sorisky A: Anti-adipogenic effect of PDGF is reversed by PKC inhibition. J Cell Physiol 2005, 204:646-653.

47. Moll UM, Slade N: p63 and p73: roles in development and tumor formation. Mol Cancer Res Mcr 2004, 2:371-386.

48. La Sala D, Macaluso M, Trimarchi C, Giordano A, Cinti C: Triggering of p73-dependent apoptosis in osteosarcoma is under the control of E2Fs-pRb2/p130 complexes. Oncogene 2003, 22:3518-3529.

49. Zhao Y, Fei C, Zhang X, Zhang Y, Guo J, Gu S, Li X, Chang C: Methylation of the p73 gene in patients with myelodysplastic syndromes: correlations with apoptosis and prognosis. Tumour Biol J Int Soc Oncodevelopmental Biol Med 2013, 34:165-172.

50. Ramadan S, Terrinoni A, Catani MV, Sayan AE, Knight RA, Mueller M, Krammer PH, Melino G, Candi E: p73 induces apoptosis by different mechanisms. Biochem Biophys Res Commun 2005, 331:713-717.

51. Hashimoto H, Hong S, Bhagwat AS, Zhang X, Cheng X: Excision of 5-hydroxymethyluracil and 5-carboxylcytosine by the thymine DNA glycosylase domain: its structural basis and implications for active DNA demethylation. Nucleic Acids Res 2012, 40:10203-10214.

52. Ruczinski I, Jorgensen TJ, Shugart YY, Schaad YB, Kessing B, Hoffman-Bolton J, Helzlsouer K, Kao WHL, Wheless L, Francis L, Alani RM, Strickland PT, Smith MW, Alberg AJ: A population-based study of DNA repair gene variants in relation to non-melanoma skin cancer as a marker of a cancer-prone phenotype. Carcinogenesis 2012, 33:1692-1698.

53. Raetz AG, Xie Y, Kundu S, Brinkmeyer MK, Chang C, David SS: Cancer-associated variants and a common polymorphism of MUTYH exhibit reduced repair of oxidative DNA damage using a GFP-based assay in mammalian cells. Carcinogenesis 2012, 33:2301-2309.

54. Zenri F, Hiroi H, Momoeda M, Tsutsumi R, Hosokawa Y, Koizumi M, Nakae H, Osuga Y, Yano T, Taketani Y: Expression of retinoic acid-related orphan receptor alpha and its responsive genes in human endometrium regulated by cholesterol sulfate. I Steroid Biochem Mol Biol 2012, 128:21-28.

55. Demidov ON, Kek C, Shreeram S, Timofeev O, Fornace AJ, Appella E, Bulavin DV: The role of the MKK6/p38 MAPK pathway in Wip1-dependent regulation of ErbB2-driven mammary gland tumorigenesis. Oncogene 2007, 26:2502-2506.

56. Satoh N, Maniwa Y, Bermudez VP, Nishimura K, Nishio W, Yoshimura M, Okita Y, Ohbayashi C, Hurwitz J, Hayashi Y: Oncogenic phosphatase Wip1 is a novel prognostic marker for lung adenocarcinoma patient survival. Cancer $S c i$ 2011, 102:1101-1106.

57. Lu X, Nguyen T-A, Moon S-H, Darlington Y, Sommer M, Donehower LA: The type 2C phosphatase Wip1: an oncogenic regulator of tumor suppressor and DNA damage response pathways. Cancer Metastasis Rev 2008, 27:123-135.

58. Cabrol A, Desruennes M, Léger P: [Monitoring of patients with cardiac transplantation]. Ann Cardiol Angéiologie 1990, 39:b607-612.

59. Kondratiev S, Gnepp DR, Yakirevich E, Sabo E, Annino DJ, Rebeiz E, Laver NV: Expression and prognostic role of MMP2, MMP9, MMP13, and MMP14 matrix metalloproteinases in sinonasal and oral malignant melanomas. Hum Pathol 2008, 39:337-343.

60. Kessenbrock K, Plaks V, Werb Z: Matrix metalloproteinases: regulators of the tumor microenvironment. Cell 2010, 141:52-67.

61. Eferl R, Wagner EF: AP-1: a double-edged sword in tumorigenesis. Nat Rev Cancer 2003, 3:859-868

62. Ma Y, Li Q, Cui W, Miao N, Liu X, Zhang W, Zhang C, Wang J: Expression of c-Jun, p73, Casp9, and N-ras in thymic epithelial tumors: relationship with the current WHO classification systems. Diagn Pathol 2012, 7:120.

63. Calin GA, Dumitru CD, Shimizu M, Bichi R, Zupo S, Noch E, Aldler H, Rattan S, Keating M, Rai K, Rassenti L, Kipps T, Negrini M, Bullrich F, Croce CM: Frequent deletions and down-regulation of micro- RNA genes miR15 and miR16 at $13 q 14$ in chronic lymphocytic leukemia. Proc Natl Acad Sci USA 2002, 99:15524-15529.

64. Linsley PS, Schelter J, Burchard J, Kibukawa M, Martin MM, Bartz SR, Johnson JM, Cummins JM, Raymond CK, Dai H, Chau N, Cleary M, Jackson AL, Carleton M, Lim L: Transcripts targeted by the microRNA-16 family cooperatively regulate cell cycle progression. Mol Cell Biol 2007, 27:2240-2252. 
65. Song J, Liu P, Yang Z, Li L, Su H, Lu N, Peng Z: MiR-155 negatively regulates C-Jun expression at the posttranscriptional level in human dermal fibroblasts in vitro: implications in UVA irradiation-induced photoaging. Cell Physiol Biochem Int I Exp Cell Physiol Biochem Pharmacol 2012, 29:331-340.

66. Hassan ZK, Elamin MH, Daghestani MH, Omer SA, Al-Olayan EM, Elobeid MA, Virk P, Mohammed OB: Oleuropein induces anti-metastatic effects in breast cancer. Asian Pac J Cancer Prev Apjcp 2012, 13:4555-4559.

67. Sutton KM, Doucette CD, Hoskin DW: NADPH quinone oxidoreductase 1 mediates breast cancer cell resistance to thymoquinone-induced apoptosis. Biochem Biophys Res Commun 2012, 426:421-426.

68. Chou R-H, Wen H-C, Liang W-G, Lin S-C, Yuan H-W, Wu C-W, Chang W-SW: Suppression of the invasion and migration of cancer cells by SERPINB family genes and their derived peptides. Oncol Rep 2012, 27:238-245.

69. Evans MK, Yu C-R, Lohani A, Mahdi RM, Liu X, Trzeciak AR, Egwuagu CE: Expression of SOCS1 and SOCS3 genes is differentially regulated in breast cancer cells in response to proinflammatory cytokine and growth factor signals. Oncogene 2007, 26:1941-1948.

70. Jamal B, Sengupta PK, Gao Z-N, Nita-Lazar M, Amin B, Jalisi S, Bouchie MP, Kukuruzinska MA: Aberrant amplification of the crosstalk between canonical Wnt signaling and N-glycosylation gene DPAGT1 promotes oral cancer. Oral Oncol 2012, 48:523-529.

71. García-Tuñón I, Ricote M, Ruiz A, Fraile B, Paniagua R, Royuela M: Interleukin-2 and its receptor complex (alpha, beta and gamma chains) in in situ and infiltrative human breast cancer: an immunohistochemical comparative study. Breast Cancer Res BCr 2004, 6:R1-7.

72. Hackanson B, Bennett KL, Brena RM, Jiang J, Claus R, Chen S-S, Blagitko-Dorfs N, Maharry K, Whitman SP, Schmittgen TD, Lübbert M, Marcucci G, Bloomfield CD, Plass C: Epigenetic modification of CCAAT/enhancer binding protein alpha expression in acute myeloid leukemia. Cancer Res 2008, 68:3142-3151.

73. Yang Z, Bian C, Zhou H, Huang S, Wang S, Liao L, Zhao RC: MicroRNA hsa-miR-138 inhibits adipogenic differentiation of human adipose tissue-derived mesenchymal stem cells through adenovirus EID-1. Stem Cells Dev 2011, 20:259-267.

74. Smith WL, DeWitt DL, Garavito RM: Cyclooxygenases: structural, cellular, and molecular biology. Annu Rev Biochem 2000, 69:145-182.

75. Méric J-B, Rottey S, Olaussen K, Soria J-C, Khayat D, Rixe O, Spano J-P: Cyclooxygenase-2 as a target for anticancer drug development. Crit Rev Oncol Hematol 2006, 59:51-64.

76. Kim J, Bagyinszky E, Chang YH, Choe G, Choi B-O, An SSA, Kim S: A novel PSEN1 H163P mutation in a patient with early-onset Alzheimer's disease: clinical, neuroimaging, and neuropathological findings. Neurosci Lett 2012, 530:109-114

77. Van Vlerken LE, Kiefer CM, Morehouse C, Li Y, Groves C, Wilson SD, Yao Y, Hollingsworth RE, Hurt EM: EZH2 is required for breast and pancreatic cancer stem cell maintenance and can be used as a functional cancer stem cell reporter. Stem Cells Transl Med 2013, 2:43-52.

78. Ren G, Baritaki S, Marathe H, Feng J, Park S, Beach S, Bazeley PS, Beshir AB, Fenteany G, Mehra R, Daignault S, AlMulla F, Keller E, Bonavida B, de la Serna I, Yeung KC: Polycomb protein EZH2 regulates tumor invasion via the transcriptional repression of the metastasis suppressor RKIP in breast and prostate cancer. Cancer Res 2012, 72:3091-3104.

79. Lee HW, Choe M: Expression of EZH2 in renal cell carcinoma as a novel prognostic marker. Pathol Int 2012, 62:735-741.

80. Banerjee R, Mani R-S, Russo N, Scanlon CS, Tsodikov A, Jing X, Cao Q, Palanisamy N, Metwally T, Inglehart RC, Tomlins S, Bradford C, Carey T, Wolf G, Kalyana-Sundaram S, Chinnaiyan AM, Varambally S, D'Silva NJ: The tumor suppressor gene rap1GAP is silenced by miR-101-mediated EZH2 overexpression in invasive squamous cell carcinoma. Oncogene 2011, 30:4339-4349.

81. Liu J, Xie Y-S, Wang F-L, Zhang L-J, Zhang Y, Luo H-S: Cytotoxicity of 5-Aza-2'-deoxycytidine against gastric cancer involves DNA damage in an ATM-P53 dependent signaling pathway and demethylation of P16(INK4A). Biomed Pharmacother Biomédecine Pharmacothérapie 2013, 67:78-87.

doi:10.1186/1742-4682-11-S1-S8

Cite this article as: El Baroudi et al.: Pathway landscapes and epigenetic regulation in breast cancer and

melanoma cell lines. Theoretical Biology and Medical Modelling 2014 11(Suppl 1):S8.

\section{Submit your next manuscript to BioMed Central and take full advantage of:}

- Convenient online submission

- Thorough peer review

- No space constraints or color figure charges

- Immediate publication on acceptance

- Inclusion in PubMed, CAS, Scopus and Google Scholar

- Research which is freely available for redistribution 